\title{
Diffuse Presence of Myeloblasts in Chronic Subdural Hematoma of a Young Adult Patient without Systemic Hematologic Disorder
}

\author{
Shota Yokoyama ${ }^{1}$ Toru Fukuhara ${ }^{1}$ Yoichiro Namba \\ ${ }^{1}$ Department of Neurological Surgery, National Hospital Organization \\ Okayama Medical Center, Okayama, Japan \\ 2 Department of Hematology and Oncology, National Hospital \\ Organization Okayama Medical Center, Okayama, Japan
}

\author{
Shoji Asakura ${ }^{2}$
}

J Neurol Surg Rep 2014;75:e129-e132.

\author{
Address for correspondence Toru Fukuhara, MD, Department of \\ Neurological Surgery, National Hospital Organization Okayama \\ Medical Center, 1711-1 Tamasu, Kita-ku, Okayama, 701-1192, Japan \\ (e-mail: torufk@ninus.ocn.ne.jp).
}

\begin{abstract}
Keywords

- chronic subdural hematoma

- erythroblast

- hematologic disorder

- myeloblast

Objective Myeloblasts are rarely found in the composition of a chronic subdural hematoma (CSH), and reported cases with myeloblasts in CSH have all been associated with systemic hematologic disorders. We present a young man with CSH manifesting the diffuse presence of myeloblasts, although no systemic hematologic disorders were identified.

Participant A 27-year-old man, complaining of a headache lasting for a few months, was diagnosed with right $\mathrm{CSH}$, and the aspirated hematoma was sent for cytological evaluation because no apparent etiologic episode was found. The diffuse presence of precursor cells, such as myeloblasts and erythroblasts, mimicking the aspirated bone marrow, was confirmed. This finding was suggestive of a systemic hematologic disorder, although the systemic evaluations were negative.

Results The patient's hematoma reaccumulated twice, and finally hematoma and enhanced dura were removed by craniotomy under general anesthesia. Further histologic evaluation did not show any precursor cells, and he has remained asymptomatic for $>2$ years without any evidence of the hematologic disorder.

Conclusion We believe this is the first case with CSH that contained myeloblasts as well as erythroblasts in an otherwise healthy patient. A possible etiology was considered for the origin of precursor cells in his CSH.
\end{abstract}

\section{Introduction}

Traumatic chronic subdural hematoma (CSH) is common in elderly patients with the peak incidence in the sixth and seventh decades of life. ${ }^{1}$ Young adults rarely have $\mathrm{CSH}$, and in this population, other etiologies including intracranial arachnoid cysts ${ }^{2}$ or spontaneous intracranial hypotension ${ }^{3}$ often coexist and promote the development of the CSH. It may also occur as a rare comorbid intracranial lesion with some invasive or metastatic malignancies. ${ }^{4,5}$

We encountered a young adult with $\mathrm{CSH}$ without an episode of head trauma. In his subdural hematoma, bone marrow cells with two elements (i.e., erythroblasts and myeloblasts) existed, although no systemic disorders were found. His CSH was somewhat refractory but eventually cured with repeated surgeries. We believe this is the first case of CSH with diffuse myeloblasts in an otherwise healthy patient, and the etiologic aspects are discussed in this article.

\section{Case Report}

A 27-year-old man visited our clinic complaining of a chronic headache lasting $>2$ months. He was previously in excellent health and denied any episodes of head trauma. His headache received

December 1, 2013

accepted after revision

March 15, 2014

published online

June 24, 2014
DOI http://dx.doi.org/

10.1055/s-0034-1376199. ISSN 2193-6358. 〔c 2014 Georg Thieme Verlag KG
Stuttgart · New York

License terms

(®) $\Theta \circledast$ 

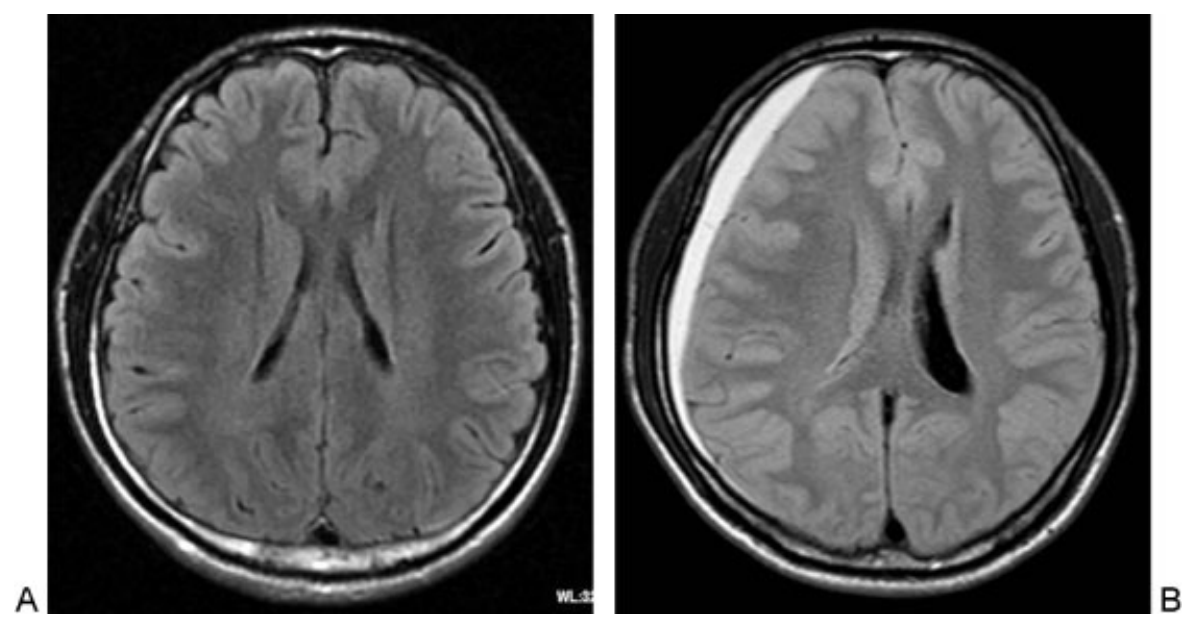

Fig. 1 Preoperative axial fluid-attenuated inversion recovery magnetic resonance images obtained (A) 1 month before presentation and (B) on admission to our hospital. (A) Although he had already complained of severe headache for a month, no abnormal findings were detected. (B) Right chronic subdural hematoma compressing the right lateral ventricle with a mild leftward midline shift was confirmed.

was mild in the beginning, but it had built up gradually during the last 2 months. He underwent magnetic resonance (MR) imaging at another hospital 1 month prior to visiting our clinic for the work-up of this headache. No abnormality was detected ( - Fig. 1A). His headache affected his daily life, and MR images were again performed at our hospital, revealing a right chronic subdural hematoma with a mild leftward midline shift (-Fig. 1B). He was admitted for surgical treatment, and drainage and irrigation of the hematoma was scheduled on the following day. His preoperative laboratory examination did not reveal any remarkable abnormalities: a white blood cell (WBC) count of $7,400 / \mathrm{mm}^{3}$ (54\% segmented neutrophils), hemoglobin $(\mathrm{Hb})$ of $16.0 \mathrm{~g} / \mathrm{dL}$, and platelet count of $268,000 / \mathrm{mm}^{3}$. He had an intact consciousness and no neurologic deficits except a severe headache. In the surgery, a burr hole was made under local anesthesia. The hematoma was aspirated and then irrigated with artificial cerebrospinal fluid (CSF). The hematoma volume was $80 \mathrm{~mL}$, a homogeneous dense liquid without any clot formation. Because this was an unusual presentation in a young adult without any predisposing events, the aspirated hematoma was sent for cytological evaluation.

After the surgery, his headache completely resolved. The cytological result of the aspirated hematoma was surprising; the finding mimicked a bone marrow aspiration (-Fig. $2 \mathbf{A}$ ). The cells consisted of myeloid and erythroid bone marrow elements, which were confirmed by myeloperoxidase staining (-Fig. 2B) and glycophorin A, respectively. Based on these findings, the tentative diagnosis was bone marrow cell migration into the subdural space; however, a hematologic disorder was suspected. A whole-body contrast computed tomography (CT) scan was performed to rule out any systemic diseases and resulted in negative findings. The hematologic profile was unremarkable, and several serum tumor markers including Amp-CML or WT1 mRNA were all within the normal range. The patient refused a bone marrow puncture. Although he was discharged 5 days after the surgery without any symptoms, a close follow-up with regular blood checks including a hematologic profile at the clinic was scheduled.
Two weeks after discharge, his headache relapsed and he visited our emergency department. His CSH had reaccumulated, confirmed by head CT scan, and emergency surgery was performed again. The hematoma was aspirated from the

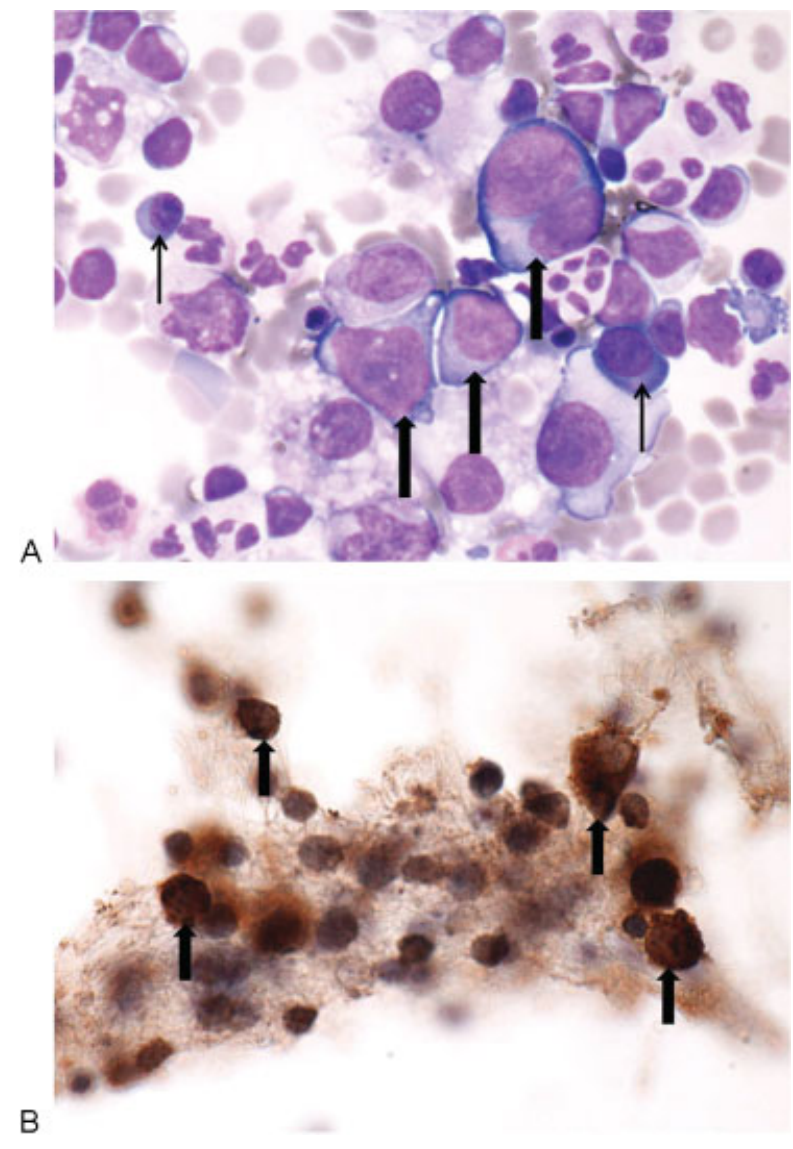

Fig. 2 Photomicrographs of the cytology specimen showing the aspirated hematoma on his first surgery. (A) Myeloblasts (large arrows) are seen in the hematoma as well as erythroblasts (small arrow). MayGiemsa staining; original magnification $\times 40$. (B) Myeloperoxidase staining indicates the diffuse presence of myeloblasts (arrows). Original magnification $\times 40$. 
same burr hole under local anesthesia. The hematoma volume was $60 \mathrm{~mL}$. The not as dense hematoma, containing xanthochromic fluid, was again sent for cytological evaluation. This time, abnormal cells were sparse in the fluid; most were inflammatory cells and necrotic cells with no precursor cells identified. The aspirated hematoma was also sent for G-band karyotyping, revealing a normal karyotype, although the examinable cells were sparse. He recalled an episode of severe transient neck pain due to chiropractic manipulation 1 month prior to the occurrence of his headache, although this neck pain did not last. Intracranial hypotension syndrome was suspected as a possible cause of the recurrent $\mathrm{CSH}$; however, radioisotope cisternography resulted in negative findings without any CSF leak in the spine. The patient did well after this repeat surgery, and he was discharged 3 days later.

His second relapse of headache occurred 2 weeks after the second surgery, and it became so severe that he was moaning in pain. MR images showed a right frontal subdural hematoma reaccumulation around the frontal portion, associated with diffuse dural and meningeal enhancement on the right (-Fig. 3A). Although the volume of subdural hematoma seemed smaller than his first presentation, no pain medication could relieve his severe headache. Meningeal irritation seemed to be responsible for this headache, and it was necessary to rule out malignant dural invasion histologically. Although digital subtraction cerebral angiography was performed to evaluate any vascular abnormalities relating to CSH accumulation, no abnormal findings such as aneurysms or vascular malformations were obtained.

Craniotomy for the dura excision and hematoma removal under general anesthesia was performed. In the operation, when the dura was exposed successively by bone flap removal, numerous bleeding points were found on the dura surface, for which complete hemostasis was difficult to obtain. The dura was incised, which was found to be thick, and then widely excised. The bleeding continued from the dura edge, and when removing the subdural hematoma, which was mostly composed of a hard clot encased by outer and inner membranes, significant hemorrhage occurred from the inner surface of the remaining dura, especially around the frontal midline. For the hemostasis, a human fibrinogen/thrombincoated collagen patch was packed into the subdural space on the frontal midline. Brain swelling occurred subsequent to hematoma removal, and then a decompressive craniectomy was performed. Gore-Tex was substituted for the widely excised dura. The removed hematoma and dura were sent for histologic analysis. The amount of bleeding during this surgery was $\sim 1,200 \mathrm{~mL}$.

After this surgery, his severe headache disappeared. Histologically, the hematoma and thickened dura revealed no malignancy; most of the hematoma was erythrocyte clots with necrotic cells and inflammatory cells, and the thickened dura revealed angiogenesis as well as inflammatory changes. No precursor cells were found in the entire specimen. Postoperative MR findings revealed brain swelling (-Fig. 3B); however, for 3 weeks after this surgery with decompressive craniectomy, his condition remained stable, and no recurrent headache occurred. Autologous cranioplasty was then performed after confirming the resolution of brain swelling. At this cranioplasty, no major bleeding occurred, and the packed human fibrinogen/thrombin-coated collagen patch was removed without any further bleeding.

After the cranioplasty, the patient remained asymptomatic and was discharged. He returned to his original life without any neurologic deficits. He did not have any difficulty in performing his job and had no recurrence of the headache. His MR images performed at 6 months after the decompressive craniectomy did not show any further abnormalities, and the meningeal enhancement on MR images had disappeared (-Fig. 3C). The hematologic evaluation was performed for 18 months, showing no evidence of systemic hematologic disorder: a WBC count of $6,100 / \mathrm{mm}^{3}$ (48\% segmented neutrophils), Hb of $16.5 \mathrm{~g} / \mathrm{dL}$, and platelet count of $252,000 / \mathrm{mm}^{3}$. The hematologist believes the patient is unlikely to develop any hematologic disorder relating to his prior $\mathrm{CSH}$, and the patient discontinues his regular hematologic follow-up. He has been healthy now for $>2$ years.
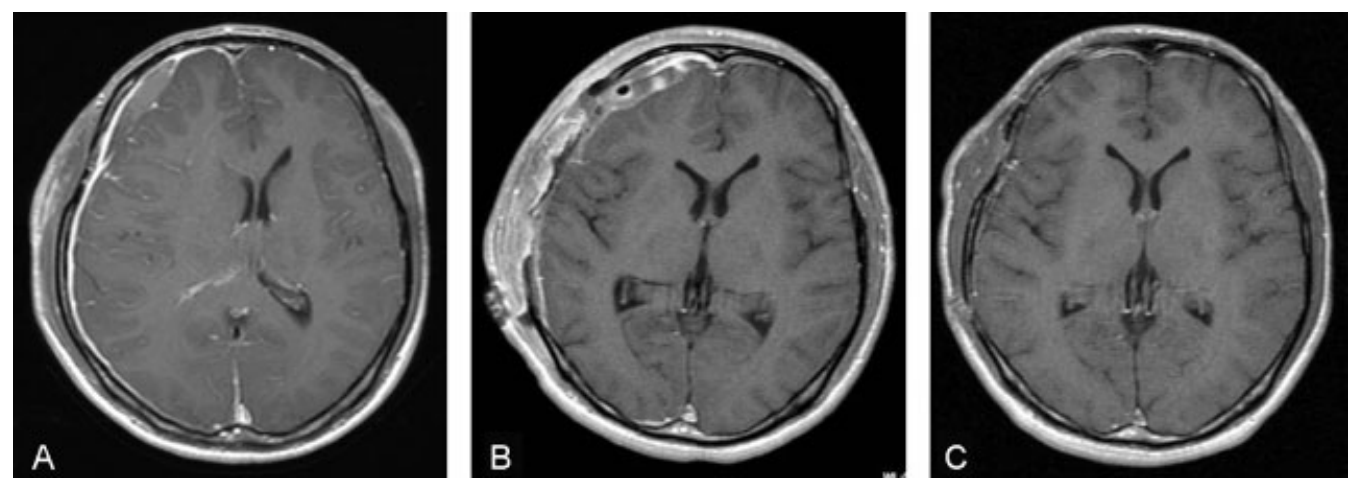

Fig. 3 Contrast-enhanced T1-weighted axial magnetic resonance images obtained (A) 3 days before, (B) 1 week after, and (C) 6 months after decompressive craniectomy. (A) The reaccumulation of the subdural hematoma was localized around the right frontal portion. In addition, the diffuse dural enhancement on the right associated with adjacent meningeal enhancement was visible. A mild leftward midline shift was observed. (B) Although midline shift was improved, the brain swelling on the right was observed over the area of decompressive craniectomy. The subdural mass around the frontal portion was considered to be the packed human fibrinogen/thrombin-coated collagen patch from the operation. (C) No abnormal dural enhancement or brain swelling was observed. 


\section{Discussion}

Aspirated CSH has been reported to consist of basically clotted erythrocyte and erythrocyte-fibrin components, ${ }^{1,6}$ although it may contain proliferating fibroblasts or inflammatory cells including macrophages with phagocytosed hemosiderin, lymphocytes, ${ }^{1}$ and eosinophils at a later stage. ${ }^{7}$ The findings of erythrocyte precursors in CSH were first described in $1966,{ }^{8}$ and one of the possible explanations was extramedullary erythropoiesis against profound anemia. However, caution was advised regarding the pathologic significance of erythrocyte precursors in CSH because erythroblasts were found in up to a third of aspirated CSHs, ${ }^{5}$ and this finding does not indicate malignancies. Rather, it merely represents the benign phenomenon against anemia or extramedullary erythropoiesis. ${ }^{1,9}$

In contrast, myeloblasts in CSH were rarely found, and reported cases have all been associated with systemic hematologic disorders such as myelofibrosis, ${ }^{10,11}$ myelodysplastic syndrome, ${ }^{12}$ and postpolycythemic myeloid metaplasia. ${ }^{13}$ Although no megakaryocytic element was found in the presented case, we believe this is the first reported case with CSH that contained myeloblasts as well as erythroblasts in an otherwise healthy patient. In the formerly reported cases, the intracranial lesions contained a nodular extra-axial mass rather than merely liquid hematoma. The uniqueness of our presented case is that the aspirated CSH was homogeneous fluid, and no nodular mass was detected on MR images. Due to the reaccumulation of hematoma, another hematoma aspiration was performed, but no precursor cells were found. We finally performed craniectomy for removal of the dura and hematoma, so detailed histologic evaluations could be carried out; however, no precursor cells were detected either. It has been a year since his last surgery, and the patient has been in a good health without any relapses. He is unlikely to have any systemic hematologic disorders, and other etiologic considerations should be made for the origin of precursor cells in his CSH.

As for the origin of the precursor cells in the subdural space, several authors suggest the theory of "leak from the bone marrow" through some unseen channel into the subdural space. ${ }^{8,10}$ It is possible for the dura to have extramedullary hematopoiesis, and it could also be the supplier of the precursor cells in the subdural space. Taking into account this theory, the patient's clinical course is possibly explained as CSH with unknown etiology. Considering his preceding headache, he may have had intracranial hypotension due to chiropractic malpractice, which initiated CSH formation, although it was undetectable and possibly resolved spontaneously. In the course of CSH formation, migration of stem cells from cranial bone marrow or dura with extramedullary hematopoiesis occurred. These migrated precursor cells may increase and affect the enlargement of CSH. After the aspira- tion in the initial operation, precursor cells were mostly removed; however, there may have existed some channels with dura and CSH capsule, and then it recurred. Certainly, the dura is prone to bleeding, although we failed to identify extramedullary hematopoiesis on the excised dura specimen.

We do not know whether the presence of precursor cells in CSH itself affects the aggressive clinical course of the patient. There may be unrealized cases with CSH containing myeloblasts that can be cured with a single burr hole surgery. Although several former reports recommended radiation therapy for CSH with myeloblasts, ${ }^{10-12}$ our single case indicates the radiation is unjustifiable without the evidence of a systemic hematologic disorder. Still, if we encounter this, it is mandatory to suspect systemic hematologic disorders and perform systemic detailed examinations.

\section{References}

1 Kuhn E, Dorji T, Rodriguez J, Rosai J. Extramedullary erythropoiesis in chronic subdural hematoma simulating metastatic small round cell tumor. Int J Surg Pathol 2007;15(3):288-291

2 Domenicucci M, Russo N, Giugni E, Pierallini A. Relationship between supratentorial arachnoid cyst and chronic subdural hematoma: neuroradiological evidence and surgical treatment. J Neurosurg 2009;110(6):1250-1255

3 Schievink WI, Maya MM, Moser FG, Tourje J. Spectrum of subdural fluid collections in spontaneous intracranial hypotension. J Neurosurg 2005;103(4):608-613

4 Cinalli G, Zerah M, Carteret M, et al. Subdural sarcoma associated with chronic subdural hematoma. Report of two cases and review of the literature. J Neurosurg 1997;86(3):553-557

5 Reichman J, Singer S, Navi B, et al. Subdural hematoma in patients with cancer. Neurosurgery 2012;71(1):74-79

6 Poljaković Z, Petrusić I, Kalousek M, Brzović Z, Jadro-Santel D. Correlative pathology of subdural hematoma with computerized tomography. Neurol Croat 1991;41(1-2):21-32

7 Sarkar C, Lakhtakia R, Gill SS, Sharma MC, Mahapatra AK, Mehta VS. Chronic subdural haematoma and the enigmatic eosinophil. Acta Neurochir (Wien) 2002;144(10):983-988; discussion 988

8 Slater JP. Extramedullary hematopoiesis in a subdural hematoma. Case report. J Neurosurg 1966;25(2):211-214

9 Firsching R, Müller W, Thun F, Boop F. Clinical correlates of erythropoiesis in chronic subdural hematoma. Surg Neurol 1990;33(3):173-177

10 Lund RE, Aldridge NH. Computed tomography of intracranial extramedullary hematopoiesis. J Comput Assist Tomogr 1984; 8(4):788-790

11 Tun K, Kaptanoglu E, Celikmez RC, Gurcan O, Turkoglu OF, Kutluay L. Meningeal extramedullary haematopoiesis mimicking subdural hematoma. J Clin Neurosci 2008;15(2):208-210

12 Di Ieva A, Aimar E, Tancioni F, et al. Focal extra-axial hemorrahagic mass with subdural hemorrhage secondary to extramedullary hematopoiesis in idiopathic myelodysplastic sindrome. J Neurosurg Sci 2007;51(1):29-32

13 Brown JA, Gomez-Leon G. Subdural hemorrhage secondary to extramedullary hematopoiesis in postpolycythemic myeloid metaplasia. Neurosurgery 1984;14(5):588-591 